\section{Evaluating Hepatitis B Seroprotection and Revaccination for Children With Inflammatory Bowel Disease}

To the Editor: We read with interest the article entitled "Serologic Status of Routine Childhood Vaccines, Cytomegalovirus, and Epstein-Barr Virus in Children With Inflammatory Bowel Disease" by deBruyn et al., particularly the section on hepatitis B (HBV) seroprotection. We conducted a similar study in our US tertiary care center, analyzing HBV seroprotection in 159 children with inflammatory bowel disease (IBD) age 0-22 who received the three-dose HBV primary series and had been exposed to a biologic agent. In our study, only $26 \%$ (41/159) of patients had HBV seroprotection [hepatitis B surface antibody (HBsAb) $\geq 10 \mathrm{IU} / \mathrm{L}]$, compared to $71 \%(82 / 115)$ of patients in the deBruyn et al. study (Table 1). Previously reported HBV seroprotection rates in pediatric patients with IBD have shown similar heterogeneity, ranging from $28 \%$ to $59 \% .^{1,2}$ The difference may be partially explained by regional variation in vaccine timing, as HBV vaccination occurs during grade school in Canada (the deBruyn study) versus infancy in the USA. Because seroprotection wanes with time, earlier vaccination may manifest as lower titers by adolescence. While the deBruyn study included patients who were not receiving biologics, our study included only patients exposed to biologics and could thus represent a more immunocompromised population. Immunocompromise

doi: 10.1093/ibd/izz095 Published online 11 May 2019

TABLE 1. Hepatitis B Surface Antibody Titers Before and After Hepatitis B Booster Vaccination in Pediatric Patients With Inflammatory Bowel Disease

\begin{tabular}{lcc}
\cline { 2 - 3 } & Inadequate Titers & Adequate Titers \\
\cline { 2 - 3 } & $\begin{array}{c}(\mathrm{HBs}<<10 \text { IU/L }) \\
(\%)\end{array}$ & $\begin{array}{c}(\mathrm{HBs} \mathrm{I} / \mathrm{L})(\%) \\
\text { IU })\end{array}$ \\
\hline After primary series $(\mathrm{n}=159)$ & $118(74)$ & $41(26)$ \\
After one HBV booster $(\mathrm{n}=33)$ & $7(21)$ & $26(79)$ \\
$\begin{array}{l}\text { After complete HBV revaccination } \\
(\mathrm{n}=59)\end{array}$ & $1(2)$ & $58(98)$
\end{tabular}

Abbreviations: HBV, hepatitis B; HBsAb, hepatitis B surface antibody.

may impair HBV immunity because of poor T-cell response, although future studies exploring T-cell mediated immune memory in pediatric patients with IBD are needed. ${ }^{3}$

After identifying patients with inadequate HBV seroprotection, it is important to consider revaccination strategies. Our group evaluated response to a single $\mathrm{HBV}$ booster by analyzing serum HBsAb titers following one booster dose. We found that, among the 33 patients who received one booster dose followed by titer recheck, $79 \%(26 / 33)$ of patients achieved seroconversion. We additionally evaluated response to complete booster vaccination, defined as receipt of three boosters or documented seroconversion before three boosters. These results showed that $98 \%(58 / 59)$ of patients achieved seroprotection after complete booster vaccination (Table 1). Overall, these data suggest that booster vaccination is effective for achieving seroconversion in this population. Adult IBD studies also suggest that nonresponders, after one HBV booster, may convert after subsequent boosters. Cossio-Gil et al. found that of 53 initial nonresponders, 53\% responded to a second booster, whereas Gisbert et al. found that of 95 initial nonresponders, $42 \%$ responded to a second booster., ${ }^{4}$ Thus, checking titers after each booster and providing further vaccines only as needed may prevent unnecessary vaccination while achieving seroprotection. Such efforts may help optimize HBV protection in children with IBD.

\section{Erica J. Brenner, MD*, Ravi Jhaveri, $\mathbf{M D}^{\dagger}$, Michael D. Kappelman, MD, MPH*, and Ajay S. Gulati, MD*}

Key Words: hepatitis B, seroprotection, revaccination, booster, pediatric, inflammatory bowel disease

*University of North Carolina Department of Pediatric Gastroenterology, Chapel Hill, North Carolina, USA; ${ }^{\dagger}$ Feinberg School of Medicine Department of Pediatric Infectious Diseases, Chicago, Illinois, USA

Supported by: The authors have no sources of support to disclose.

Address correspondence to: Erica J. Brenner, MD, 333 S. Columbia Street, 247 MacNider Hall CB \#7229, Chapel Hill, NC 27514 (Erica.Brenner@) unchealth.unc.edu).

\section{REFERENCES}

1. Watts A, Bennett WE, Molleston JP, et al. Incidence of low seroimmunity to hepatitis B virus in children with inflammatory bowel disease. J Pediatr Gastroenterol Nutr. 2017;65:551-554.

2. Yeo SJ, Lee HS, Jang BI, et al.; Crohn's and Colitis Association in Daegu-Gyeongbuk (CCAiD). Nonimmunity against hepatitis B virus infection in patients newly diagnosed with inflammatory bowel disease. Intest Res. 2018;16:400-408

3. Banatvala JE, Van Damme P. Hepatitis B vaccine - do we need boosters? J Viral Hepat. 2003;10:1-6.

4. Cossio-Gil Y, Martínez-Gómez X, CampinsMartí M, et al. Immunogenicity of hepatitis B vaccine in patients with inflammatory bowel disease and the benefits of revaccination. $J$ Gastroenterol Hepatol. 2015;30:92-98.

5. Gisbert JP, Villagrasa JR, RodríguezNogueiras A, et al. Efficacy of hepatitis B vaccination and revaccination and factors impacting on response in patients with inflammatory bowel disease. Am J Gastroenterol. 2012;107:1460-1466. 